\title{
ECOLOGIA DOMESTICA DE LA TRIPANOSOMIASIS AMERICANA: PERFIL AIIMENTARIO DEL TRIATOMA INFESTANS EN UN AREA RURAL DE I.A PROVINCIA DE SANTIAGO DEL ESTERO, ARGENTINA
}

\author{
Cristina Wisnivesky-Colli ${ }^{1}$, A. M. Ruiz², O. Ledesma ${ }^{3}$, R. E. Gütler1, \\ Marta Lauricella2, D.O. Salomón², Nora Solarz ${ }^{1}$ y Elsa L. Segura1
}

\begin{abstract}
Se describen los resultados entomológicos de un estudio sobre transmisión del Tripanosoma cruzi realizado en un caserio rural de Argentina. Se determinó la prevalencia de infección y el perfil alimentario de los Triatoma infestans domiciliados, relacionándose su densidad con el tipo de vivienda. Seis de las 18 viviendas estudiadas eran nuevas o mejoradas y en 16 los habitantes aplicaban hexaclorociclohexano. La menor densidad de vinchucas correspondió a las casas higiénicas o mejoradas con tratamiento quimico. Los ranchos tratados presentaron un número de insectos 10 veces menor que un rancho no tratado, indicando la efectividad del control autogestado. Se hallaron T. infestans en el $100 \%$ de los dormitorios pero en sólo el $53 \%$ de los locales peridomiciliarios. Un 39\% de las vinchucas intradomiciliarias estaban infectadas. El $96 \%$ de los insectos de dormitorios se alimentaron sobre hombre, perro, pollo y gato, siendo el indice afinidad para el perro 13 veces superior al correspondiente al hombre. Se discute la importancia epidemiológica de la concentración de vectores dentro de los dormitorios y su asociación trófica con los perros convivientes, asi como la necessidad de combinar la mejora de vivienda con educación sanitaria.
\end{abstract}

Palabras claves: Triatominos. Perfil alimentario. Control. Transmisión de T. cruzi.

La tripanosomiasis americana se transmite a través de una red compleja formada por numerosos elementos bióticos y abióticos que posibilitan su existencia y perpetuación en la naturaleza. El estudio integral de los factores biológicos que intervienen en la cadena epidemiológica (humanos, mamiferos domésticos, vectores y el Trypanosoma cruzi) es fundamental para comprender la mecánica de la transmission y diseñar estrategias dirigidas a controlarla.

La mayoría de los estudios epidemiológicos realizados en poblaciones humanas de la Argentina 3 -5 8917 estuvieron dirigidos principalmente a investigar la existencia de infección y enfermedad en seres humanos, sin integrar dentro de un mismo panorama factores relacionados con el vector, con los

1. Unidad Ecología de Parásitos, Dep. Ciências Biológicas, Fac. Ciências Exactas y Naturales, Univ. de Buenos Aires. Ciudad Universitaria, Pab. II, 49 piso, 1428 Buenos Aires, Argenina (dirección para correspondencia).

2. Instituto de Diagnóstico e Investigación de la Enfermedad de Chagas, "Dr. Mario Fatala Chabén", Buenos Aires.

3. Centro de Enfermedades y Patologia Regional, Hospital Independencia, Santiago del Estero.

Este trabajo se realizó con un subsidio del Programa Nacional de Enfermedades Endémicas, Secretaria de Ciencia y Tecnologia.

Recebido para publicaçăo em 25/11/85. reservorios domésticos, con el tipo de vivienda y con las medidas de lucha de los pobladores contra el vector. Con el objetivo de obtener un enfoque ecológico integral de la transmisión doméstica del $T$. cruzi hemos realizado una serie de encuestas epidemiológicas en caserios rurales del norte de Argentina. En ellas se estudió la prevalencia del $T$. cruzi en las poblaciones humanas, de mamíferos domiciliarios y de vectores, tomando como nexo de referencia el perfil alimentario de los triatominos para analizar las relaciones entre los distintos elementos de la cadena epidemiológica. En el presente trabajo se describen los resultados entomológicos de un estudio ecológico realizado en un caserio rural, La Invernada, en la provincia de Santiago del Esterc.

\section{MATERIALES Y METODOS}

Descripción del área de estudio

La localidad elegida, denominada La Invernada, está situada en el Departamento de Figueroa, a $100 \mathrm{~km}$ de la capital de la Provincia de Santiago del Estero dentro de la Región Chaqueña Occidental de Argentina ${ }^{6}$ (Fig. 1). La formación vegetal característica es el bosque xerófilo sin solución de continuidad con esteras halófilas, como resultado de la degradación del monte de quebracho original (Schinopsis 
Wisnivesky-Colli C, Ruiz AM, Ledesma O, Gütler RE, Lauricella M, Salomón DO, Solarz N, Segura EL. Ecologia domestica de la tripanosomiasis americana: perfil alimentario de Triatoma infestans en un area rural de la Provincia de Santiago del Estero, Argentina. Revista da Sociedade Brasileira de Medicina Tropical 20: 31-39, Jan-Mar, 1987.

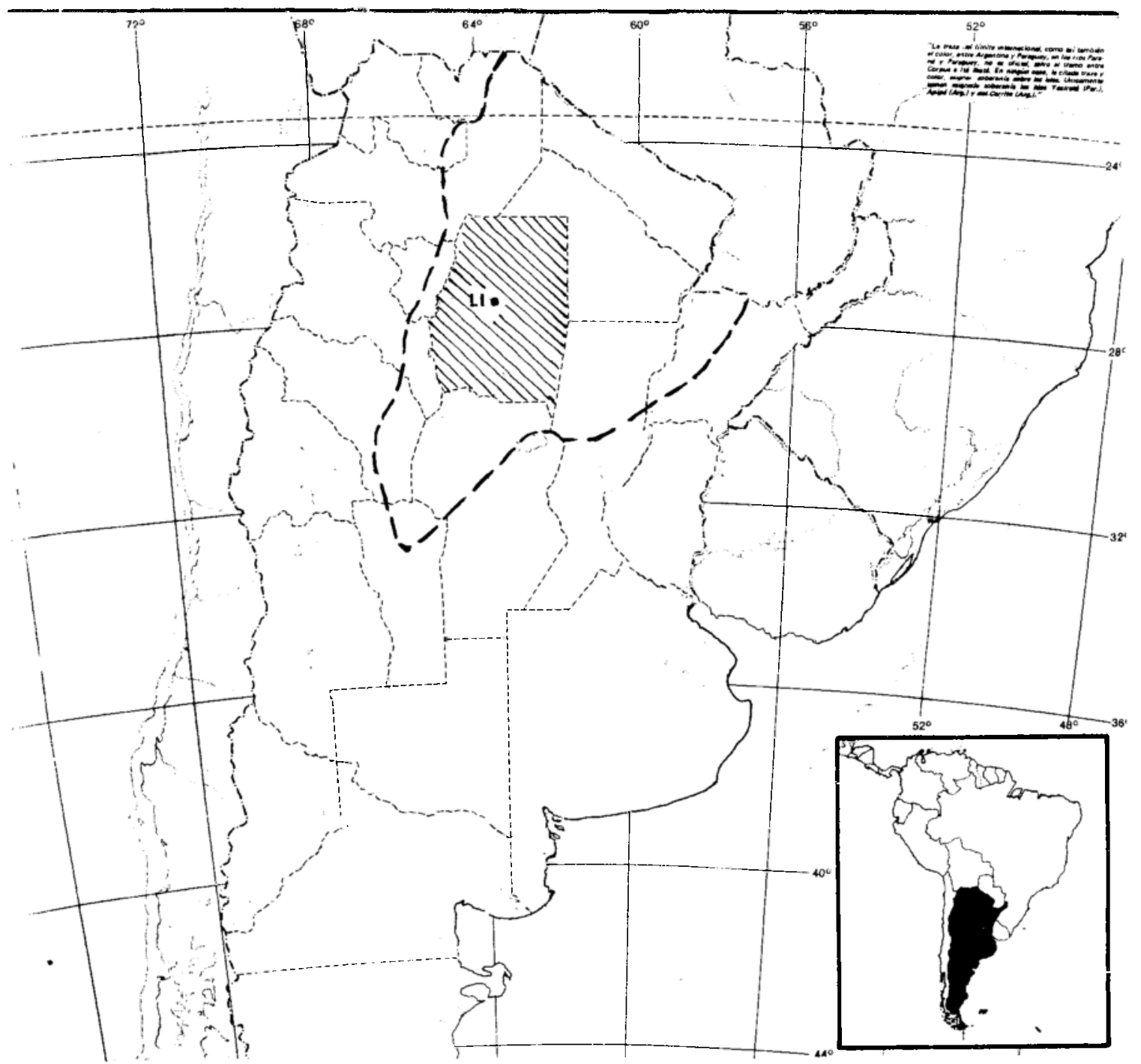

Fig. 1. Mapa de la región central y norte de Argentina, indicando la Región Fitogenética Chaqueña (línea contínua) y la localidad de La Invernada en la Provincia de Santiago del Estero, donde se realizó este estudio.

lorentzii). El clima es seco con precipitaciones de 500 a $800 \mathrm{~mm}$ y temperaturas medias en el mes más cálido de $28^{\circ} \mathrm{C}$ y en el más frio de $14,6^{\circ} \mathrm{C}$.

El caserio, formado por aproximadamente 143 viviendas se halla situado a lo largo de dos rutas provinciales asfaltadas. Para realizar el estudio se seleccionaron al azar 20 casas ( $14 \%$ del total), en una superficie de aproximadamente $100 \mathrm{~km}^{2}$. El Ministro de Salud Pública provincial estaba llevando a cabo um plan de mejora de la vivienda que se había iniciado 3 años antes de la realización de este trabajo. Este hecho determinaba la existência de 3 tipos distintos de viviendas: a) ranchos típicos, con techo de paja y troncos de arbustos intercalados con tierra, paredes de palo a pique o de adobe (ladrillos de barro sin cocer) revestidas con barro y piso de tierra (Fig. 2a);

b) viviendas higiénicas con techos de chapa de zinc o de fibrocemento y paredes de ladrillos y argamassa, con piso de cemento (Fig. 2b);

c) viviendas mejoradas com paredes o techos modificados y piso de tierra.

La estructura del domicilio era similar a la descripta para outras zonas de la Región Chaqueña ${ }^{23}$. La casa estaba constituida por 2 cuartos (dormitorios) y una galería delantera o alero. Los anexos peridomici- 
Wisnivesky-Colli C, Ruiz AM, Ledesma O, Gütler RE, Lauricella M, Salomón DO, Solarz N, Segura EL. Ecologia domestica de la tripanosomiasis americana: perfil alimentario de Triatoma infestans en un area iural de la Provincia de Santiago del Estero, Argentina. Revista da Sociedade Brasileira de Medicina Tropical 20: 31-39, Jan-Mar, 1987.

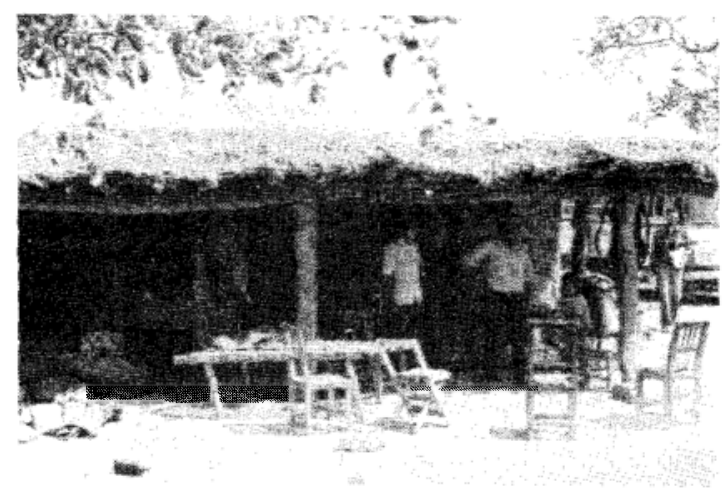

Fig. 2 a - Rancho tipico en La Invernada, Provincia de Santiago del Estero, Argentina.

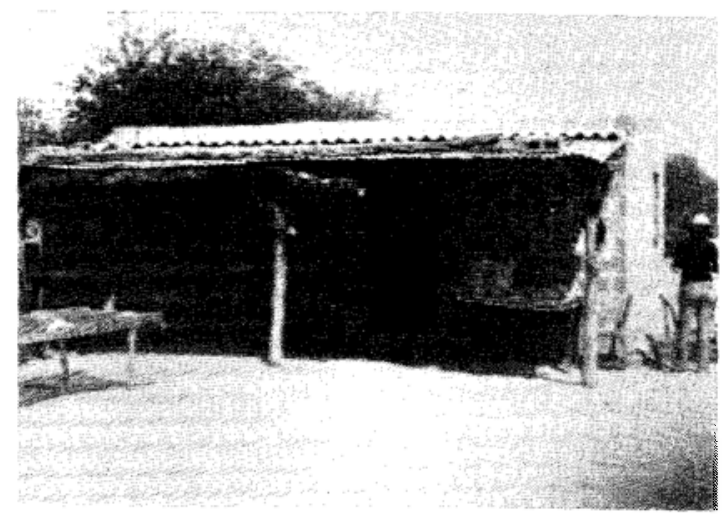

Fig. 2 b - Vivienda higiénica en La Invernada, Provincia de Santiago del Estero, Argentina.

liarios, situados en un radio de 5 a $60 \mathrm{~m}$ de la vivienda, estaban constituídos por el depósito y la cocina, construídos en forma similar a la vivienda, y el corral. El depósito era utilizado para guardar materiales diversos (aperos, herramientas, etc ) y frecuentemente dormían allí animales domésticos.

El caserio habia sido rociado sistemáticamente con insecticidas en una campaña gubernamental desarrollada en la década de los años 60 y que posteriormente fue interrumpida en 1971. Cuando se realizó este estudio, el único tratamiento químico era de tipo domiciliario, ya que los pobladores quemaban pastillas de hexaclorociclohexano con periodicidades variables entre 1 y 3 veces por año.

Todos los habitantes, los perros, gatos y aves de corral de las 20 viviendas fueron censados en septiembre de 1980. Se completó una encuesta epidemiológica en cada vivienda que permitió obtener algunos datos socioeconómicos y culturales de la población.

\section{Muestreo y examen de las vinchucas}

La captura de los triatominos se realizó dentro de las viviendas y en los anexos peridomiciliarios, utilizando movilizadores químicos (neopinamín al $0,2 \%$ en isobutano) para provocar la salida de los triatominos de sus refugios, efectuándose simultáneamente la revisión sistemática de los enseres domésti$\cos$. El equipo de colectores estaba integrado por 3 personas que trabajaban simultáneamente durante 1 hora en cada local (esfuerzo de captura $=\mathbf{3}$ horas/ hombre).

\section{RESULTADOS}

\section{Caracteristicas habitacionales e infestación}

Dos de las 20 viviendas seleccionadas fueron eliminadas del estudio por hallarse sus habitantes temporariamente ausentes. Entre las 18 casas estudiadas sólo 2 eran viviendas higiénicas de reciente construcción, 12 eran ranchos típicos, y 4 casas mejoradas. En cuanto a la antigüedad de las viviendas, la mayoría (14/18) tenían entre 3 y 9 años de construídas, 3 tenían menos de 2 años y 2 más de 9 años.

En la Tabla 1 se presenta la distribución de los perros, gatos y aves de corral según el tipo de vivienda con el objeto de observar la posible influencia de la mejora de vivienda sobre la cantidad y tipo de animales más importantes para la transmisión domiciliaria de $T$. cruzi, o bien para el mantenimiento de las poblaciones del vector. No se observan diferencias en cuanto al número promedio de gallinas o de gatos presentes. Las 6 viviendas higiénicas o mejoradas presentan un mayor número de perros que los ranchos típicos.

Se calculó un número promedio de 6,5 personas tanto para los ranchos como para el resto de las viviendas. La encuesta epidemiológica permitió establecer que en todas las casas los habitantes dormían la mayor parte del año bajo el alero, salvo durante los breves periodos de frío (julio). Los perros descansaban siempre en la galeria y en algunas casas se explicitó que dormían bajo las camas. Con respecto a los gatos, la información recogida indicó que lo hacían dentro de los dormitorios, sobre las camas u outros enseres, o en las cocinas y depósitos, especialmente en los meses más frios. El patrón fue homogéneo para todas las casas, independientemente del tipo de vivienda, con excepción de una casa mejorada donde se señaló que gatos y perros dormian siempre en el depósito. Esta observación se confirmó por un lado en el perfil alimentario de las vinchucas coleccionadas en los dormitorios que presentó ingestas sobre humano y pollo exclusivamente, y por otro en el de los insectos provenientes de depósito con abundantes alimentaciones sobre perro y gato.

Con respecto a la relación entre tipo de vivienda, aplicación doméstica de insecticidas e infestación, 
Wisnivesky-Colli C, Ruiz AM, Ledesma O, Gütler RE, Lauricella M, Salomón DO, Solarz N, Segura EL. Ecologia domestica de la tripanosomiasis americana: perfil alimentario de Triatoma infestans en un area rural de la Provincia de Santiago del Estero, Argentina. Revista da Sociedade Brasileira de Medicina Tropical 20: 31-39, Jan-Mar, 1987.

Tabla 1 -Distribución de los animales domésticos según el tipo de vivienda. La Invernada, Santiago del Estero, 1980.

\begin{tabular}{lccc}
\hline $\begin{array}{c}\text { Tipo de vivienda } \\
\text { (No examinado) }\end{array}$ & $\begin{array}{c}\text { Número promedio de animales } \\
\text { por casa }\end{array}$ \\
\hline & Perros & Gatos $\begin{array}{l}\text { Gallinas, patos } \\
\text { y pavos }\end{array}$ \\
\hline $\begin{array}{c}\text { Ranchos } \\
(12)\end{array}$ & 2,4 & 0,9 & 18,5 \\
$\begin{array}{l}\text { Viviendas } \\
\text { higiénicas } \\
(2)\end{array}$ & 4 & 0,5 & 15 \\
$\begin{array}{l}\text { Viviendas } \\
\text { mejoradas } \\
(4)\end{array}$ & 3,3 & 1 & 13,3 \\
$\begin{array}{l}\text { Total de } \\
\text { animales }\end{array}$ & 50 & 16 & 305 \\
\hline
\end{tabular}

el escaso número de viviendas en las que los pobladores no realizaban tratamiento quimico, impide una buena comparación entre ranchos y casas mejoradas. En 10 de los 11 ranchos, existía uso doméstico de insecticidas y el número promedio de vinchucas colectadas por casa fue $9,3 \pm 2,4$ insectos. En el rancho no tratado se capturaron en cambio, 60 ejemplares de $T$. infestans. Sólo en una de las 4 casas mejoradas los moradores no usaban insecticidas, colectándose 26 vinchucas; en las 3 restantes, contrastantemente, el número promedio de insectos por casa fue sensiblemente inferior $(\bar{X}=2,7 \pm 1,2)$. En las 2 casas higiénicas existentes se aplicaban insecticidas detectándose en promedio $1,5 \pm 0,5$ vinchucas por casa (datos no presentados en tablas).

El $100 \%$ de los dormitorios estaban infestados mientras que el $53 \%$ de los locales peridomiciliarios albergaban insectos, y de ellos, los depósitos eran los locales más frecuentemente positivos. En sólo 3 de las 13 cocinas examinadas $(23,1 \%)$ se hallaron ejemplares de $T$. infestans (datos no presentados en tablas).

\section{Infección por $\mathrm{T}$. cruzi en los ejemplares $\mathrm{de} \mathrm{T}$. infestans} coleccionados

En la Tabla 2 se presentan los datos de la distribución por sitios de colección de los insectos capturados en las 18 viviendas, según su estadío. La captura total (355 ejemplares) se dividió en un $96 \%$ entre los dormitorios y los depósitos, siendo mayor en los primeros. En las cocinas la muestra coleccionada fue pequeña y constituída exclusivamente por ninfas $\mathrm{V}$ y adultos. No se hallaron triatominos en los corrales. Un $61 \%$ del total de vinchucas capturadas eran ninfas, con un franco predominio del IV y $\mathrm{V}$ estadio. El número de machos y hembras hallado fue similar. $\mathrm{La}$ tasa de infección de los $T$. infestans examinados fue del $33 \%$, variando según el sitio de colección entre el 26 y el 39\%. Se observó un incremento en el porcentaje global de $T$. infestans infectados en función

Tabla 2 - Distribución de la infección por $\mathrm{T}$. cruzi por estadio y sitio de colección de los ejemplares de $\mathrm{T}$. infestans colectados en La Invernada, Santiago del Estero, 1980.

\begin{tabular}{|c|c|c|c|c|c|c|c|c|c|c|c|c|c|c|}
\hline \multirow[b]{3}{*}{$\begin{array}{l}\text { Sitio de } \\
\text { colección }\end{array}$} & \multicolumn{14}{|c|}{ Estadio } \\
\hline & \multicolumn{2}{|r|}{$I-I I$} & \multicolumn{2}{|r|}{$I I I$} & \multicolumn{2}{|r|}{$I V$} & \multicolumn{2}{|r|}{$V$} & \multicolumn{2}{|c|}{ Hembras } & \multicolumn{2}{|r|}{ Machos } & \multicolumn{2}{|r|}{ Total } \\
\hline & $\begin{array}{l}\text { No } \\
\text { col. }\end{array}$ & $\begin{array}{c}\text { No } \\
\text { Pos/exam. } \\
\text { (\%) }\end{array}$ & $\begin{array}{l}\text { No } \\
\text { col. }\end{array}$ & $\begin{array}{c}\text { No } \\
\text { Pos/exam. } \\
\text { (\%) }\end{array}$ & $\begin{array}{l}\text { No } \\
\text { col. }\end{array}$ & $\begin{array}{c}\text { No } \\
\text { Pos/exam } \\
(\%)\end{array}$ & $\begin{array}{l}\text { № } \\
\text { col. }\end{array}$ & $\begin{array}{c}\text { No } \\
\text { Pos/exam } \\
\text { (\%) }\end{array}$ & $\begin{array}{l}\text { No } \\
\text { col. }\end{array}$ & $\begin{array}{c}\text { No } \\
\text { Pos/exam } \\
(\%)\end{array}$ & $\begin{array}{l}\text { No } \\
\text { col. }\end{array}$ & $\begin{array}{c}\text { No } \\
\text { Pos/exam } \\
(\%)\end{array}$ & $\begin{array}{l}\text { No } \\
\text { col. }\end{array}$ & $\begin{array}{c}\text { No } \\
\text { Pos/exam } \\
(\%)\end{array}$ \\
\hline Dormitorios & 14 & $\begin{array}{l}0 / 6 \\
(0)\end{array}$ & 17 & $\begin{array}{l}1 / 15 \\
(7)\end{array}$ & 36 & $\begin{array}{l}6 / 28 \\
(21)\end{array}$ & 42 & $\begin{array}{c}12 / 36 \\
(33)\end{array}$ & 44 & $\begin{array}{c}28 / 44 \\
(64)\end{array}$ & 40 & $\begin{array}{c}18 / 39 \\
(46)\end{array}$ & 193 & $\begin{array}{c}65 / 168 \\
(39)\end{array}$ \\
\hline Depósitos & 8 & $\begin{array}{l}0 / 3 \\
(0)\end{array}$ & 15 & $\begin{array}{c}0 / 12 \\
(0)\end{array}$ & 48 & $\begin{array}{l}8 / 43 \\
(19)\end{array}$ & 45 & $\begin{array}{c}18 / 42 \\
(43)\end{array}$ & 17 & $\begin{array}{l}6 / 17 \\
(35)\end{array}$ & 13 & $\begin{array}{c}1 / 12 \\
(8)\end{array}$ & 146 & $\begin{array}{c}33 / 129 \\
(26)\end{array}$ \\
\hline Cocinas & 1 & $\begin{array}{l}0 / 1 \\
(0)\end{array}$ & 0 & & 0 & & 8 & $\begin{array}{l}3 / 8 \\
(37)\end{array}$ & 3 & $\begin{array}{c}1 / 3 \\
(33)\end{array}$ & 4 & $\begin{array}{l}1 / 3 \\
(33)\end{array}$ & 16 & $\begin{array}{l}5 / 15 \\
(33)\end{array}$ \\
\hline $\begin{array}{l}\text { Total } \\
(\%)\end{array}$ & $\begin{array}{l}23 \\
(6)\end{array}$ & $\begin{array}{c}0 / 10 \\
(0)\end{array}$ & $\begin{array}{l}32 \\
(9)\end{array}$ & $\begin{array}{c}1 / 27 \\
(4)\end{array}$ & $\begin{array}{c}84 \\
(24)\end{array}$ & $\begin{array}{c}14 / 71 \\
(20)\end{array}$ & $\begin{array}{c}95 \\
(27)\end{array}$ & $\begin{array}{c}33 / 86 \\
(38)\end{array}$ & $\begin{array}{c}64 \\
(18)\end{array}$ & $\begin{array}{c}35 / 64 \\
(55)\end{array}$ & 57 & $\begin{array}{c}20 / 54 \\
(37)\end{array}$ & 355 & $\begin{array}{c}103 / 312 \\
(33)\end{array}$ \\
\hline
\end{tabular}


Wisnivesky-Colli C, Ruiz AM, Ledesma O, Gütler RE, Lauricella M, Salomón DO, Solarz N, Segura EL. Ecologia domestica de la tripanosomiasis americana: perfil alimentario de Triatoma infestans en un area rural de la Provincia de Santiago del Estero, Argentina. Revista da Sociedade Brasileira de Medicina Tropical 20: 31-39, Jan-Mar, 1987.

del estadío, detectándose tripanosomas a partir de las ninfas III. En los depósitos se registró un porcentaje significativamente mayor de ninfas de $\mathrm{V}$ estadio que de adultos infectados $\left(X^{2}=4,879, p<0,05\right)$. No se hallaron diferencias importantes entre los porcentajes de machos y hembras infectados, salvo en los coleccionados en depósitos (35\% hembras positivas para T. cruzi vs $8 \%$ machos positivos).

\section{Perfil de alimentación de las vinchucas}

El análisis del perfil de alimentación que se resume en la Tabla 3, fue realizado considerando el número total de ingestas identificadas provenientes de un hospedador determinado, tanto si se trataba de identificaciones puras (sobre una sola especie de hospedador) como múltiples (acompañadas de sangre de otros hospedadores).

En la muestra total de insectos, el hospedador identificado con mayor frecuencia fue el pollo $(32,5 \%$ de las ingestas) seguido del perro $(25,0 \%)$ y el hombre $(24,2 \%)$ con porcentajes muy similares. Se halló una menor proporción de alimentaciones sobre gato $(10,8 \%)$ y animales de corral (agregado: $7,2 \%)$. No se identificaron ingestas sobre animales silvestres peridomicilarios, tales como armadillos, didélfidos o cávidos. La única comida sobre roedor fue hallada en una vinchuca de dormitorio, por lo cual podría tratarse de un roedor domiciliario. El perfil de alimentación varió en cada sitio de colección. En las muestras provenientes de los dormitorios, el $96 \%$ de las identificaciones se repartieron entre el hombre, el perro, el pollo y el gato, con predominio de las ingestas de origen humano $(38,7 \%)$ sobre las de origen canino $(25,3 \%)$. El pollo fue el hospedador predominante en las muestras coleccionadas en los depósitos, seguido por el perro, que pasó a ser el hospedador más frecuentemente identificado en los contenidos de las vinchucas de las cocinas.

Se halló un $46 \%$ de las alimentaciones sobre más de un tipo de hospedador (múltiples) en las muestras provenientes de todos los sitios de colección (Tabla 4). Un 7,6\% de las vinchucas coleccionadas en los dormitorios se había alimentado sobre 4 ó 5 hospedadores diferentes. El mayor procentaje de alimentaciones múltiples fue hallado en los ejemplares de $T$. infestans capturados en las cocinas.

Los indices de afinidad*11,18 de los insectos provenientes de todos los sitios de colección (Tabla 5) arrojaron un valor máximo para el gato, seguido del pollo y del perro. El indice de afinidad para el hombre fue 13 veces inferior al índice de afinidad para el perro.

\section{DISCUSSION}

Dado que el plan de mejora de vivienda aplicado en el caserío estudiado fue parcial, no es posible evaluar el efecto de esta medida sobre la infestación domiciliaria, ya que muy pocas casas fueron efectivamente modificadas. Sin embargo, los datos obtenidos parecen indicar que la sola sustitución de paredes y techos no excluye la colonización por parte de los triatominos, cuando persisten viviendas precarias infestadas en el poblado. De alli la importancia de combinar la modificación de las habitaciones humanas con el uso sistemático de insecticidas, en áreas endémicas.

El uso doméstico de insecticidas tiene un efecto importante sobre la densidad de insectos por casa, puesto que en los ranchos donde los pobladores realizaban tratamiento químico el número promedio

Tabla 3 - Perfil de alimentación de T. infestans, discriminadas por sitio de colección. La Invernada, Stgo. del Estero, 1980.

\begin{tabular}{|c|c|c|c|c|c|c|c|c|c|}
\hline $\begin{array}{l}\text { Sitio de } \\
\text { colección }\end{array}$ & Total de ingestas & & N.o $y$ & de ingesto & eactivas & te $a$ los $a$ & ntos inmu & ueros & \\
\hline & & Pollo & Perro & Humano & Gato & Cabra & Caballo & Cerdo & Roedor \\
\hline Dormitorio & 230 & $\begin{array}{c}45 \\
(19,6)\end{array}$ & $\begin{array}{c}58 \\
(25,3)\end{array}$ & $\begin{array}{c}89 \\
(38,7)\end{array}$ & $\begin{array}{c}28 \\
(12,2)\end{array}$ & $\begin{array}{c}2 \\
(0,9)\end{array}$ & $\begin{array}{c}6 \\
(2,6)\end{array}$ & $\begin{array}{c}1 \\
(0,4)\end{array}$ & $\begin{array}{c}1 \\
(0,4)\end{array}$ \\
\hline Depósito & 138 & $\begin{array}{c}77 \\
(55,8)\end{array}$ & $\begin{array}{c}28 \\
(20,3)\end{array}$ & $\begin{array}{c}5 \\
(3,6)\end{array}$ & $\begin{array}{c}9 \\
(6,5)\end{array}$ & $\begin{array}{c}17 \\
(12,3)\end{array}$ & $\begin{array}{c}0 \\
(0,0)\end{array}$ & $\begin{array}{c}2 \\
(1,4)\end{array}$ & $\begin{array}{c}0 \\
(0,0)\end{array}$ \\
\hline Cocina & 20 & $\begin{array}{c}4 \\
(20,0)\end{array}$ & $\begin{array}{c}11 \\
(55,0)\end{array}$ & $\begin{array}{c}0 \\
(0,0)\end{array}$ & $\begin{array}{c}5 \\
(25,0)\end{array}$ & $\begin{array}{c}0 \\
(0,0)\end{array}$ & $\begin{array}{c}0 \\
(0,0)\end{array}$ & $\begin{array}{c}0 \\
(0,0)\end{array}$ & $\begin{array}{c}0 \\
(0,0)\end{array}$ \\
\hline Total & 388 & $\begin{array}{c}126 \\
(32,5)\end{array}$ & $\begin{array}{c}97 \\
(25,0)\end{array}$ & $\begin{array}{c}94 \\
(24,2)\end{array}$ & $\begin{array}{c}42 \\
(10,8)\end{array}$ & $\begin{array}{c}19 \\
(4,9)\end{array}$ & $\begin{array}{c}6 \\
(1,5)\end{array}$ & $\begin{array}{c}3 \\
(0,8)\end{array}$ & $\begin{array}{c}1 \\
(0,2)\end{array}$ \\
\hline
\end{tabular}

\footnotetext{
* Indice de afinidad $=\frac{\text { No de vinchucas alimentadas sobre el Hospedador X }}{\text { Biomasa en } \mathrm{Kg} \text { del Hospedador X presente }}$
} 
Wisnivesky-Colli C, Ruiz AM, Ledesma O, Güller RE, Lauricella M, Salomón DO, Solarz N, Segura EL. Ecologia domestica de la tripanosomiasis americana: perfil alimentario de Triatoma infestans en un area rural de la Provincia de Santiago del Estero, Argentina. Revista da Sociedade Brasileira de Medicina Tropical 20: 31-39, Jan-Mar, 1987.

Tabla 4 - Número y (\%) de ejemplares de $\mathbf{T}$. infestans alinentados sobre 1, 2 o más hospedadores, discriminados por sitio de colección. La Invernada, Stgo. del Estero, 1980.

\begin{tabular}{|c|c|c|c|c|c|}
\hline \multirow[t]{2}{*}{$\begin{array}{l}\text { Sitio de } \\
\text { colección }\end{array}$} & \multirow{2}{*}{$\begin{array}{c}\text { No de insectos } \\
\text { con ingestas } \\
\text { identificadas } \\
(\%)\end{array}$} & \multicolumn{4}{|c|}{$\begin{array}{c}\text { No hospedadores identificados en } \\
\text { cada ingesta } \\
\text { (\%) }\end{array}$} \\
\hline & & 1 & 2 & 3 & 40 más \\
\hline Dormitorios & $\begin{array}{c}132 \\
(93,6)\end{array}$ & $\begin{array}{c}67 \\
(50,8)\end{array}$ & $\begin{array}{c}44 \\
(33,3)\end{array}$ & $\begin{array}{c}11 \\
(8,3)\end{array}$ & $\begin{array}{l}10^{* *} \\
(7,6)\end{array}$ \\
\hline Depósitos & $\begin{array}{c}91 \\
(93,8)\end{array}$ & $\begin{array}{c}55 \\
(60,4)\end{array}$ & $\begin{array}{c}26 \\
(28,6)\end{array}$ & $\begin{array}{c}9 \\
(9,9)\end{array}$ & $\begin{array}{c}1 \\
(1,1)\end{array}$ \\
\hline Cocinas & $\begin{array}{c}13 \\
(92,9)\end{array}$ & $\begin{array}{c}6 \\
(46,2)\end{array}$ & $\begin{array}{c}7 \\
(53,8)\end{array}$ & 0 & 0 \\
\hline Total & $\begin{array}{c}236 \\
(93,7)\end{array}$ & $\begin{array}{c}128 \\
(54,2)\end{array}$ & $\begin{array}{c}77 \\
(32,6)\end{array}$ & $\begin{array}{c}20 \\
(8,5)\end{array}$ & $\begin{array}{c}11 \\
(4,7)\end{array}$ \\
\hline $\begin{array}{l}\text { Tentitica } \\
\text { lizados; } \\
\text { analizado } \\
* * \text { 8 ejempla }\end{array}$ & & & 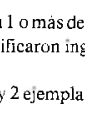 & 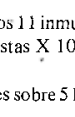 & \\
\hline
\end{tabular}

Tabla 5 - Valores medios y desviaciones standard de los indices de la afinidad para el hombre y los animales domésticos de los ejemplares de $\mathrm{T}$. infestans coleccionados en 11 viviendas *. La Invernada, Stgo. del Estero, 1980.

\begin{tabular}{lcc}
\hline Hospedador & \multicolumn{2}{c}{ Índice de afinidad** } \\
& $X^{*}$ (media) & SD (desviación standard) \\
Gato & 2,0 & 1,6000 \\
Pollo & 0,67 & 0,7030 \\
Perro & 0,33 & 0,3580 \\
Cabra & 0,072 & 0,0982 \\
Humano & 0,025 & 0,0331 \\
Cerdo & 0,004 & 0,0014 \\
\hline
\end{tabular}

* Se consideraron sólo las viviendas con un nivel de infestación superior a 10 ejemplares de $T$. infestans.

*o* Indice de afinidad $=\frac{\text { No de vinchucas alimentadas sobre el hospedador } x}{\text { Biomasa en } \mathrm{Kg} \text { de hospedador } \mathrm{x} \text { presente }}$

de $T$. infestans colectado fue sensiblemente menor ( 3,1 vinchucas/hora/hombre) que en el mismo tipo de vivienda no tratada con insecticidas (20 vinchucas/ hora/hombre). La amplia difusión de la aplicación doméstica de $\mathrm{HCH}$ entre los habitantes podría deberse al efecto combinado de las campañas gubernamentales y de la acción de los agentes sanitarios. Resulta así evidente que una acción educativa, realizada dentro de una campaña de control coherente y sistemática, con participación de la comunidad, posiblemente redun- daría en la interrupción de la transmisión de la enfermedad, como ha ocurrido en otros paises de América ${ }^{15}$.

La existencia de mejores condiciones habitacionales, no parece afectar otros aspectos de la forma de vida de los habitantes, tales como sus hábitos de reposo y su relación con los animales domésticos. Tanto en los ranchos como en las casas nuevas o mejoradas las gentes dormían la mayor parte del año en la galería. Por otra parte en las casas higiénicas y mejoradas se detectó el mismo número de personas, gatos y gallinas que en los ranchos, mientras que el número de perros convivientes fue ligeramente mayor. Estos animales guardaban el mismo estrecho contacto con las personas en los ranchos que en las casas modificadas, ya que la mejora de la casa habitación no implicó una mejora del peridomicilio (construcción de gallineros y de casillas para perros) ni modificó la costumbre de cohabitar con los animales domésticos, especialmente los perros. Estas observaciones señalan la importancia de combinar todo programa de mejora de vivienda con campañas de educación sanitaria en la comunidad.

La mayor abundancia de $T$. infestans en dormitorios y depósitos señala la estrecha asociación ecológica entre estos insectos y la típica construcción de la vivienda rancho. En este estudio, las capturas por hora/hombre dentro de las habitaciones fueron inferiores con respecto a las halladas en una zona rural de Córdoba ${ }^{23} 24$. Sin embargo, es preciso tener en cuenta que el muestreo entomológico de este trabajo se realizó a mediados de septiembre, al final de un invierno riguroso, mientras que los datos de Córdoba corresponden al mes de diciembre, en pleno verano, y la densidad poblacional de los triatominos sufre variaciones estacionales marcadas ${ }^{20}$. Aún asi, las densidades de problación existentes seriam suficientes para asegurar una transmisión activa del $T$. cruzi, como lo revelaría la existencia de un $29 \%$ de niños de 0 a 4 años infectados (Wisnivesky-Colli, en preparación), a pesar de que los datos entomológicos puntuales no permiten inferir las densidades de vinchucas presentes en el pasado.

El corral típico de esta zona, con su empalizada de troncos separados entre sí, no resulta apto para la colonización de triatominos y difiere de los de otras zonas del pais, en los cuales, los cercos de ramazón y guano albergan abundantes poblaciones de vinchu$\operatorname{cas}^{162223}$

Los porcentajes globales de infección por $T$. cruzi en los $T$. infestans coleccionados en los dormitorios son inferiores a los señalados para otras zonas endémicas de Argentina ${ }^{8} 23$. Sin embargo, las altas proporciones de $\mathrm{V}$ y adultos infectados, que son aquellos estadíos que requieren ingestas más abundan- 
Wisnivesky-Colli C, Ruiz AM, Ledesma O, Gütler RE, Lauricella M, Salomón DO, Solarz N, Segura EL. Ecologia domestica de la tripanosomiasis americana: perfil alimentario de Triatoma infestans en un area rural de la Provincia de Santiago del Estero, Argentina. Revista da Sociedade Brasileira de Medicina Tropical 20: 31-39, Jan-Mar, 1987.

tes o más numerosas, respectivamente, confirman la existencia de un importante flujo de parásitos circulando dentro de las viviendas. Vale la pena señalar aqui, la conveniencia de considerar las tasas de infección por $T$. cruzi en los estadíos más avanzados, en lugar de los porcentajes globales, como indicador del nivel de transmisión, cuando se realizan estudios epidemiológicos.

El predominio de ingestas sobre humanos en los ejemplares de $T$. infestans de dormitorios, contrasta con los datos obtenidos para la misma especie en Guanaco Muerto, Córdoba ${ }^{23}$, donde la principal fuente de alimentación fue el perro $(48,6 \%$ del total de comidas) y el hombre ocupó el tercer lugar en el perfil trófico $(11,9 \%)$. Si bien el volumen de alimentaciones sobre perro es importante en este estudio y similar al hallado para humanos, corresponde aproximadamente a la mitad del hallado en la localidad cordobesa. Otro tanto ocurre con el pollo como fuente de alimentación que ocupó el segundo lugar en importancia en Guanaco Muerto y pasó al tercer lugar en La Invernada. Dado que el tamaño de ambas muestras analizadas es similar, es posible que estas diferencias puedan atribuirse a distinta disponibilidad de los hospedadores dentro de la vivienda, o a diferencias en los hábitos de reposo, que requ:ariría de un estudio más detallado.

El perfil de alimentación señala claramente que aún cuando la mayor parte de las ingestas provienen de los hospedadores residentes en el sitio de colección, existe cierta movibilidad entre el domicilio y los locales peridomiciliarios. Asi lo indican las siguientes observaciones: a) Un 3,9\% de las identificaciones de los insectos de los dormitorios correspondieron a animales de corral y un porcentaje similar de ingestas sobre humanos se detectó en las provenientes de los depósitos. En la muestra de vinchucas coleccionadas en estos últimos locales, $13,7 \%$ de las comidas provenian de cabras y cerdos; b) Los porcentajes de vinchucas infectadas en dormitorios y depósitos fueron similares, si bien en los depósitos predominaron las ingestas sobre pollos; c) El porcentaje de comidas mixtas, indicador de la movilidad de las poblaciones de vinchucas 1 , fue muy alto, hallándose hasta un $8 \%$ de los insectos con ingestas provenientes de 4 ó 5 hospedadores diferentes. En este punto nuevamente los resultados de este estudio contrastan con los de Córdoba antes mencionados 23 , donde tanto el elevado porcentaje de alimentaciones puras halladas $(71,1 \%)$, como la baja contribución de los animales de corral a la alimentación de insectos del dormitorio $(0,8 \%$ del total de identificaciones), señalan una menor movilidad de las poblaciones domiciliarias y peridomiciliarias.

La estrecha asociación entre las poblaciones de $T$. infestans y los perros, se evidencia no sólo en el elevado número de ingestas sobre estos animales sino principalmente en el índice de afinidad (IA) de las vinchucas para los perros que es 13 veces superior al hallado para humanos, confirmando resultados anteriores 11,23 . Este índice se calculó midiendo la oferta de hospedadores como biomasa $(\mathrm{kg})$ como fuera sugerido por Minter ${ }^{12}$ y Rossell ${ }^{18}$ y puede ser considerado un buen indicador del grado de contacto entre hospedadores y vectores. Sin embargo es preciso realizar experimentos que permitan acotar el índice de afinidad para un intervalo apropiado de las variables que lo componen. Así el alto IA hallado para gatos, en este trabajo, deriva probablemente de la combinación de un bajo número de ingestas sobre gato y el escaso número de felinos presentes en la vivienda. El hecho que estos felinos sean también animales nocturnos, desacredita la existencia de una asociación trófica estrecha entre gatos y vinchucas domiciliarias. Por otra parte, a pesar de que estos felinos presentan altas tasas de infección en la zona estudiada, su asociación con el ciclo doméstico de $T$. cruzi, está relativizada por sus hábitos vagabundos 25 . La presencia de perros con altas tasas de infección (67\%) y de parasitemia (46\%) dentro de los dormitorios de esta comunidad 25 constituye el factor principal que sustenta y mantiene la transmisión doméstica del $T$. cruzi, como ya fuera señalado para otras regiones del pais ${ }^{19} 25$. Por otra parte, los perfiles de alimentación de $T$. infestans obtenidos en este trabajo no aportan evidencias que involucren a los animales silvestres peridomiciliarios en la cadena epidemiológica doméstica de esta zona, como se ha demonstrado para otras regiones de América $^{2} 1426$.

\section{SUMMARY}

Entomological aspects of an ecological study on domestic $\mathrm{T}$. cruzi transmission carried out in a rural community of Argentina are described. T. cruzi infection rates and the feeding profiles of domiciliary $\mathrm{T}$. infestans were determined, and bug density was related to house construction. Six of 18 houses studied were new and reformed while 16 households hexachlorocyclohexane $(\mathrm{HCH})$ had been used by the inmates. The lowest bug densities were found in new houses or those receiving good, insecticide treatment. Insecticide treated units had an average number of bugs 10 times lower (3.1 bugs/man-hour) than those found in a non treated ones indicating the effectiveness of selfpromoted control. All bedrooms but only 53\% of peridomestic structures harboured bugs. T. cruzi infection was detected in $39 \%$ of domiciliary. $\mathrm{T}$. infestans $96 \%$ of the domiciliary bugs (96\%) fed on man, dog, chicken and cat. The affinity index for dog was 13 times higher than that for man. The importance of the high density of $\mathrm{T}$. infestans bugs in 
Wisnivesky-Colli C, Ruiz AM, Ledesma O, GǘterRE, Lauricella M, Salomón DO, Solarz N, Segura EL. Ecologia domestica de la tripanosomiasis americana: perfil alimentario de Triatoma infestans en un area rural de la Provincia de Santiago del Estero, Argentina. Revista da Sociedade Brasileira de Medicina Tropical 20: 31-39, Jan-Mar, 1987.

bedrooms and their close association with "roommate" dogs is discussed in relation to transmission as well as the need to combine house improvernent and health education programmes.

Key Words: Triatomines, Feeding profile. Control. $\Upsilon$. cruzi transmission.

\section{AGRADECIMIENTOS}

Este trabajo se realizó con la valiosa colaboración de las seguintes instituciones y personas: Servicio Provincial de Lucha (Santiago del Estero), Sres. Dido Lopez y Alberto Velázquez; Dirección de Atención Primaria de la Salud (Santiago del Estero), à trãvés de los supervisores y agentes sanitarios de La Irrvernada; Sra. Magdalena de Deffis (INDIECH) y Sr. Domingo Fernández (INDIECH), Dres. Amalia Marteleur y Gustavo Barbieri (Centro de Enfermedades y Patología Regional, Santiago del Estero).

\section{REFERENCIAS BIBLIOGRÁFICAS}

1. Barreto MP. Estudos sobre reservatórios e vectores silvestres do Trypanosoma cruzi. XXXI. Observaçōes sobre a associação entre reservatónios e vectores com especial referencia a regiäo nordeste do Estado dé São Paulo. Revista Brasileira de Biologia 28:481-494, 1968.

2. Barreto MP. Possible role of wild mammals and triatomines in the transmission of Trypanosoma cruzi to man. In: New Approaches in American Trypanosomiasis Research, Washington, PAHO. Scientific Publication № 318: 307-316, 1976.

3. Blaksley JC, Carcavallo RV. La enfermedad de ChagasMazza en la Argentina I. Estudios entomoepidemiølógicos y clínicos. Ministerio de Bienestar Social, Buenos Aires, 1968.

4. Bonet $\mathrm{AH}_{4}$ Cichero JA. Estudio epidemiológico sobre la enfermedad de Chagas-Mazza en comunidades rurales de la Provincia de Córdoba. La Semana Médica $133: 581-587,1968$

5. Borda CE, Rea MJF. Ser̃oepidemiología de la enfermedad de Chagas en Yaciretá -- Apipé, Argentina. Boletin de la Oficina Sanitaria Panamericana 90: 311 $325,1981$.

6. Cabrera AL. Fitugeografia de la República Argentina Boletín de la Sociedad Argentina de Botánica 14:1-50, 1971.

7. Carcavallo RV, Plencovich AR. La enfennedad de Chagas en la Provincia de Buenus Aires, Argentiía. Boletín de la Oficina Sanitaria Panamericana 74:281-289, 1973.

8. Carcavalio RV. Algunos aspectos de la epidemiología de la enfermedad de Chagas en Venezuela y Argentina. In: New Approaches in Americaĩ Trypanosomiasis Research, Washington, PAHO. Scieritific Publication No 318:347.358, 1976.

9. Cichero JA, Bonet AH, Grand JC, Rossm AJ, Segura EL. Investigación de la prevalencia de la enfermedad de Chagas-Mazza en comunidades rurales. II Jomadas
Entomoepidemiológicas Argentinas (1965) 2:11-19, 1967.

10. Marsden PD, Virgens D, Magalhães I, Tavares-Neto J, Ferreira R, Costa CH, Castro CN, Macêdo V, Prata A. Ecología doméstica do Triatoma infestans em Mambai, Goiás, Brasil. Revista do Instituto de Medicina Tropical de São Paulo 24:364-373, 1982.

11. Mayer HF, Alcaraz IL. Estudios relacionados con las fuentes alimentarias de Triatoma infestans (Hemiptera, Reduviidae). Anales del Instituto de Medicina Regional, Tucumán 4: 195-201, 1955.

12. Minter DM. Feeding patterns of some triatomine vectors species. In: New Approaches in American Trypanosomiasis Research, Washington, PAHO. Scientific Publication No 318:33-47, 1976.

13. Minter DM. Effect on transmission to man of the presence of domestic animals in infested households. In: New Approaches in American Trypanosomiasis Research, Washington, PAHO. Scientific Publication No $318: 330-338,1976$.

14. Pifano FC. La epidemiologia de la enfermedad de Chagas en Venezuela. Archivos Venezolanos de Medicina Tropical y Parasitología Médica 5: 170-184, 1973.

15. Pinto Días JC, Borges Días R. Las viviendas y la lucha contra los vectores de la enfermedad de Chagas en el hombre, en el Estado de Minas Gerais, Brasil. Boletín de la Oficina Sanitaria Panamericana 93:453-465, 1982.

16. Ronderos RA, Schnack JA, Mauri RA. Resultados preliminares respecto a la ecologia de Triatoma infestans (Klug) y especies congenéricas con referencia especial a poblaciones peridomiciliarias. Medicina (Buenos Aires) 40 (Supl. 1): 187-196, 1980.

17. Rosembaum MB, Cerisola JA. Encuesta sobre enfermedad de Chagas en el norte de Córdoba y sur de Santiago del Estero. Prensa Médica Argentina 44:2713-2727, 1957.

18. Rossell Reyes OJ. Evaluación de la transmissión de la enfermedad de Chagas en dos caserios del Estado de Guárico (Venezuela) sometidos a rociamiento. Trabajo presentado ante la Universidad de Los Andes, Dep. dé Biologia, Facultad de Ciencias. Copia mimeografiada, 1977.

19. Ruiz AM, Wisnivesky-Colli C, Gütler R, Lazzari J, Bujas MA, Segura EL. Infección por Trypanosoma cruzi en humianos, perros y cabras en áreas rurales de la Provincia de Córdoba. Medicina (Buenos Aires) 45:539$546,1985$.

20. Schofield CJ. Density regulation of domestic populations of Triatoma infestans in Brazil. Transactions of the Royal Society of Tropical Medicine and Hygiene 74:761-769, 1980.

21. Sokal RR, Rohlf FJ. Biometry. The principles and practice of statistics in biological research. WH Freeman and Co. San Francisco, USA, 1969.

22. Soler CA, Knez NR, Neffem LE. Focos peridomésticos. Publicación del Servicio Nacional de Chagas, Jurisdicción La Rioja. 1977.

23. Wisnivesky-Colli C, Gürtler RE, Solarz N, Ruiz AM. Feeding patterns of Triatoma infestans (Hemiptera, Reduviidae) in relation to transmission of American Trypanosomiasis in Argentina. Joumal of Medical Entomology 19:645-654, 1982 
Wisnivesky-Colli C, Ruiz AM, Ledesma O, Gütler RE, Lauricella M, Salomón DO, Solarz N, Segura EL. Ecologia domestica de la tripanosomiasis americana: perfil alimentario de Triatoma infestans en un area rural de la Provincia de Santiago del Estero, Argentina. Revista da Sociedade Brasileira de Medicina Tropical 20: 31-39, Jan-Mar, 1987.

24. Wisnivesky-Colli C. Dinámica de la transmisión de la enfermedad de Chagas en la vivienda rural. Tesis de doctorado. Universidad de Buenos Aires, Argentina, 1983.

25. Wisnivesky-Colli C, Gürtler RE, Solarz ND, Lauricella M, Segura EL. Epidemiological role of humans, dogs and cats in the transmission of Trypanosoma cruzi in a central area of Argentina. Revista do Instituto de Medicina Tropical de São Paulo 27:346-352, 1985.

26. Zeledón R, Solano G, Burstin L, Swartzwelder JC. Epidemiological pattern of Chagas disease in an endemic area of Costa Rica. American Journal of Tropical Medicine and Hygiene 24:214-225, 1975. 